Discussion We found that the learning environment and selfimprovement in terms of the sense of skill retention regarding mental health activities differed according to years of experience, and it appeared that at each career stage, there are characteristics in the learning environment/self-improvement required to improve the sense of skill retention.

\section{SENSE OF DIFFICULTY, SKILL RETENTION, AND LEARNING STRATEGIES IN WORKPLACE MENTAL HEALTH ACTIVITIES BY OCCUPATIONAL HEALTH NURSES (1ST REPORT)}

Noriko Nishikido*, Mako Ishikawa, Satoko Shimamoto. Graduate School of Nursing, Tokai University, Kanagawa, Japan

\subsection{6/oemed-2018-ICOHabstracts. 1011}

Purpose To clarify the sense of difficulty in mental health activities by occupational health nurses, as well as the characteristics of skills retention and future learning tasks.

Methods Self-administered questionnaire surveys were conducted. A cluster analysis was performed of 36 items regarding mental health activities, which were classified into categories. The years of experience were divided into 5 levels, i.e. 0-4 (novice), 5-9 (early-mid), 10-14 (late-mid), 15-19 (pre-management), and 20 or more years (late management), then the responses were compared between the 5 levels.

Results Mental health activities were classified into 6 categories, including the 'construction of relationships of trust between workers and the manager, and data collection', 'assessment and support of individual consultations', 'support for job reinstatement and cooperation with relevant individuals within and outside of the enterprise', 'provision of information to workplace groups and organisations', 'construction of a mental health support system as a workplace organisation', and 'support for cases that are difficult to manage'. As the years of experienced increased, the number of nurses who had difficulty in their activities decreased. However, even in the management level, there was a strong sense of difficulty in 'support for cases that are difficult to manage'. The sense of retaining skills increased with the increase in experience. With regards to future learning tasks, for all levels of experience many nurses indicated 'support for cases that are difficult to manage'. Novices indicated 'assessment and support of individual consultations'.

Discussion Suggestions for training measures according to each career level were obtained. Among novices, training is needed for the 'assessment and support of individual consultations', while for nurses in their early- to mid-career or after, 'support for cases that are difficult to manage' as well as focusing on group and organisational support was considered appropriate.
571

CONSTRUCTION OF OCCUPATIONAL HEALTH NURSING DIAGNOSIS SYSTEM FOR GROUP AND ORGANISATION IN MENTAL HEALTH IN JAPAN

${ }^{1} \mathrm{Y}$ Yamaguchi*, ${ }^{2} \mathrm{M}$ Ito, ${ }^{3} \mathrm{~F}$ Akimoto, ${ }^{3} \mathrm{~K}$ Hara, ${ }^{3} \mathrm{~K}$ Masuzawa, ${ }^{4} \mathrm{M}$ Ito, ${ }^{4} \mathrm{H}$ Nakamur, ${ }^{4} \mathrm{M}$ Yanai, Murakami ${ }^{5},{ }^{6} \mathrm{~A}$ Nagano, ${ }^{7} \mathrm{M}$ Seto, ${ }^{8} \mathrm{R}$ Yoshida, ${ }^{9} \mathrm{~K}$ Kono, ${ }^{10} \mathrm{~A}$ Chiba, ${ }^{11} \mathrm{~N}$ Ochiai, ${ }^{12} \mathrm{M}$ Tachikawa, ${ }^{13} \mathrm{~K}$ Inomata. ${ }^{1}$ Sophia University, Tokyo, Japan; ${ }^{2}$ Tokyo Health Care University, Tokyo, Japan; ${ }^{3}$ Nippon Telegraph and Telephone East Corporation, Tokyo, Japan; ${ }^{4}$ Occupational Health Nursing Systematisation Consideration Committee in JAOHN, Fukuoka, Japan Tokyo; ${ }^{5}$ Metro Co., Ltd., Tokyo, Japan; ${ }^{6}$ Suzuki Motor Corporation, Shizuoka, Japan; ${ }^{7}$ LOIN Corporation Health Support Office (Hirai), Tokyo, Japan; ${ }^{8}$ Epson Sales Japan Corp., Tokyo, Japan; ${ }^{9}$ Research Centre for Occupational Health Nursing, Yokkaichi Nursing and Medical Care University, Mie, Japan; ${ }^{10}$ Aomori University of Health and Welfare, Aomori, Japan; ${ }^{11}$ The University of Shimane, Shimane, Japan; ${ }^{12}$ Ube Frontier University, Yamaguchi. Japan; ${ }^{13}$ Teikyo Heisei University, Tokyo, Japan

\subsection{6/oemed-2018-ICOHabstracts. 1012}

Introduction In order to conduct effective occupational health nursing $(\mathrm{OHN})$ services to groups and organisations, it is necessary to do assessment, planning, implementation and evaluation in line with the nursing process continuously. For that, systematisation of $\mathrm{OHN}$ diagnosis is necessary so that the three processes of planning (nursing diagnosis, nursing goal, nursing care) can be systematically utilised in particular. The purpose of this study is to develop OHN diagnosis (diagnostic index, related factor), nursing goal, nursing care in the mental health for groups and organisations, taking 'chronic high stress state' as an example.

Methods Development was advanced by 7 OHN researchers and $10 \mathrm{OHN}$ practitioners, receiving supervision, referring NANDA-I nursing diagnosis and Arizona University NIC. 'Chronic high stress state' which is one of nursing diagnosis in mental health in OHN, an OHN science system was constructed in a format systematically showing three processes.

Result In the nursing diagnosis name 'chronic high stress state', examples of diagnostic indicators were the bad results in stress survey at workplace and the number of workers on leave due to mental disorders, Examples of relevant factors are the qualitative burden of work, the quantitative burden of work. In addition, the nursing goal showed that mental health disorder decreased, new incidences of mental health disorder decreased, nursing care showed recommendations to managers, recommendations to health and safety committee, and so on.

Discussion By systematically arranging the three processes of 'chronic high stress state', the problems of group and organisation (nursing diagnosis and diagnostic index) and its factors (related factor), targeted form (nursing goal), appropriate nursing support, these relationships became clear. In addition, 'Chronic High Stress Condition' is considered to be the basis for constructing a highly versatile system with high support needs in the development of OHN diagnostic system in mental health. 


\section{FAMILY NURSING APPROACHES IN OCCUPATIONAL HEALTH NURSING: A LITERATURE REVIEW}

${ }^{1}$ Shio Tsuda*, ${ }^{2}$ Hisae Nakatani, ${ }^{2}$ Akiko Kanefuji, ${ }^{3}$ Mari Karikawa. ${ }^{1}$ Department of Community Health Nursing, Tokyo Medical and Dental University, Tokyo, Japan; ${ }^{2}$ Institute of Biomedical and Health Sciences, Hiroshima University, Hiroshima, Japan; ${ }^{3}$ Graduate School of Biomedical and Health Sciences, Hiroshima University, Hiroshima, Japan

\subsection{6/oemed-2018-ICOHabstracts. 1013}

Introduction Workers and work environment are changing as a result of demographic, economic, social and technological changes. Considering that the healthcare issue among workers are currently extending further beyond individuals and their families, it is necessary to examine the practice with family nursing approaches for workers in occupational health settings.

The purpose of this literature review was to explore the practice with family nursing approaches in occupational health nursing.

Methods Relevant articles from PubMed and the Cumulative Index to Nursing and Allied Health Literature were identified using the key words 'family,' and 'occupational health nursing,' published from 2000 to 2016. 77 articles were reviewed. 6 met inclusion criteria and were included for final review.

Result Six research articles have been published from 2005 to 2013. Three papers were focused on mental health issues of workers while others focused on working mothers physical activities, immigrant workers, and personal safety.

Discussion Several family related practice in occupational health nursing have been identified, while it revealed that findings were limited due to a lack of studies. The review highlighted the need to specifically study the healthcare issues of workers related family.

\section{RELATIONSHIP BETWEEN ORGANISATIONAL RESOURCES AND OCCUPATIONAL STRESS AMONG JAPANESE NURSES}

Yumi Wakida*, Kosuke Mafune*, Yusuke Noguchi*, Haruka Ido*, Ayako Hino*, Hisanori Hiro*. University of Occupational and Environmental Health, Japan, Kitakyushu, Japan

\subsection{6/oemed-2018-ICOHabstracts.1014}

Introduction Several studies have shown that occupational stress is low in workplaces where organisational resources are high. On the other hand, there are few studies that have specialised in investigating similar matters in health care workers. This study was conducted to examine whether the relationship between organisational resources and occupational stress can also be observed in Japanese nurses.

Methods The subjects were 851 nurses and midwives who were enrolled in two general hospitals as of 2015. The investigation period was July 2015 to August 2015. There were 727 respondents. Missing items were invalidated and excluded from the analysis. The independent variables were defined as WIN, age (20-50 years old), gender, and workplace (university hospital and branch hospital) and the dependent variables were the four subscales of the JCQ (job demands, job control, social support from the supervisor, and social support from coworkers). Multivariate analysis of variance was performed. WIN was used as a measure of organisational resources, which were classified into the three groups of (1) low score $(-1.0$
$S D$ or less), (2) medium score (-1.0 SD to $+1.0 S D)$, and (3) high score (+1.0 SD or more).

Results There was a significant association between all groups of WIN and job requirement, job control, supervisor's support, and colleague's support $(F(2,569)=15.35, \mathrm{p}<0.001, F$ $(2,562)=9.85, \mathrm{p}<0.001, F(2,568)=135.90, \mathrm{p}<0.001$, and $F$ $(2,565)=50.74, \mathrm{p}<0.001$ respectively)

Discussion The nurses working in two general hospitals in Japan provided similar results to those in previous studies. However, this research was limited to only two hospitals, and the same may not be found for nurses in general. In future, it may be necessary to collaborate with other institutions to target more nurses in medical institutions.

\section{FUTURE OCCUPATIONAL HEALTH NURSING ROLES}

${ }^{1}$ Susan A Randolph*, ${ }^{2} \mathrm{~K}$ Davies*. 'University of North Carolina - Chapel Hill, USA; ${ }^{2}$ Department of Defense, Pretoria, South Africa

10.1136/oemed-2018-ICOHabstracts. 1015

Aim of special session The role of the occupational health nurse $(\mathrm{OHN})$ is often defined by the workplace setting and needs of the employer. Components of the OHN role include health promotion, prevention of injury and illness, and protection of workers from health and safety hazards while using an interdisciplinary approach. Future roles of the OHN will be presented within these contexts through a global perspective.

\section{8a FUTURE OCCUPATIONAL HEALTH NURSING ROLES}

A Drummond*. UCD Centre for Safety and Health at Work, University College Dublin, Ireland

\subsection{6/oemed-2018-ICOHabstracts. 1016}

Introduction The role of Occupational Health Nurses (OHNs) depends on many factors, including legislation, population, geography, history, culture, industrial profile of hazards and risks, and on how occupational health (and safety) relates to the national health system. In most countries, occupational health services emerged towards the end of the 19th Century following the Industrial Revolution, and the stage of development of occupational services varies, with corresponding variation in the OHN role. Notwithstanding this, the role is well defined in the context of other members of the multidisciplinary team. Major changes to the world of work at the end of the 20th Century, including changing demographics, new ways of working and the rise of chronic diseases including mental health issues, are impacting occupational health in the $21 \mathrm{st}$ Century, and, with an increasing pace of change, has implications for the future role of occupational health nursing.

Methods A review of relevant literature.

Results The functions of the OHN can be tracked over time, require a wide range of skills and encompass numerous roles. Interdisciplinary and inter-professional collaboration is important. Changes are emerging in many countries that re-orientate the focus to the health of the working age population, and is not confined to those exposed to work-related risk or even to those at work. While there is a public health dimension, occupational health professionals see themselves as well placed, trained, and experienced to play a key role in this development. 